\title{
Telemonitoramento da Insuficiência Cardíaca - A Experiência de um Centro
}

\author{
Telemonitoring in Heart Failure - A Single Center Experience \\ Isabel O. Cruz, ${ }^{10}$ Susana Costa, ${ }^{2}$ Rogério Teixeira, ${ }^{2}$ Fátima Franco, ${ }^{2}$ Lino Gonçalves ${ }^{2}$ \\ Departamento de Medicina Interna, Hospital Pedro Hispano, ${ }^{1}$ Matosinhos - Portugal \\ Departamento de Cardiologia, Centro Hospitalar e Universitário de Coimbra, ${ }^{2}$ Coimbra - Portugal
}

\section{Resumo}

Fundamento: A evolução natural da insuficiência cardíaca é uma pior progressiva e internações hospitalares recorrentes. São necessárias estratégias para se detectar descompensações em tempo hábil. $O$ uso do telemonitoramento da insuficiência cardíaca é inconsistente.

Objetivos: Este estudo tem o objetivo de avaliar o impacto desse programa de telemonitoramento (PTM) em internações hospitalares e admissões em serviços de emergência.

Métodos: Este é um estudo retrospectivo observacional que analisou dados de todos os pacientes que se cadastraram no PTM de janeiro a 2018 a dezembro de 2019. Foram coletados dados demográficos, clínicos e relacionados ao PTM. O número de internações hospitalares e admissões em serviços de emergência do ano anterior e posterior ao cadastro foram comparados, utilizando-se o teste de Wilcoxon. Um p-valor bilateral de $<0,05$ foi considerado significativo.

Resultados: Um total de 39 pacientes foram cadastrados, com uma média de idade de $62,1 \pm 14$ anos e predominância de pacientes do sexo masculino (90\%). As causas mais comuns de insuficiência cardíaca foram cardiomiopatia isquêmica e dilatada. A fração de ejeção média foi de $30 \%$ e o tempo mediano da duração da doença foi de 84 meses (FIQ 33-144). Pacientes que foram cadastrados por menos de um mês foram excluídos, com um total de 34 pacientes analisados. Os pacientes foram acompanhados no PTM por um período mediano de 320 dias. O número de admissões em serviços de emergência foi reduzido em $66 \%(p<0,001)$ e o número de internações hospitalares por insuficiência cardíaca foi reduzido em $68 \%(p<0,001)$. O PTM não teve impacto no número de internações hospitalares por outras causas.

Conclusões: Este estudo sugere que o PTM poderia reduzir a utilização de serviços de saúde em pacientes com insuficiência cardíaca.

Palavras-chave: Insuficiência Cardíaca/fisiopatologia; Telemonitoramento; Hospitalização; Serviços de Emergência.

\footnotetext{
Abstract

Background: The natural history of heart failure is a progressive decline and recurrent hospital admissions. New strategies to timely detect decompensations are needed. The use of telemonitoring in heart failure is inconsistent.
}

Objectives: This study aimed to evaluate the impact of this telemonitoring program (TMP) in hospitalizations and emergency department admissions.

Methods: This is a retrospective observational study, that analyzed data of all the patients who enrolled in the TMP program from January 2018 to December 2019. Demographic, clinical, and TMP-related data were collected. The number of hospitalizations and emergency department admissions from the year before and after enrollment were compared, using the Wilcoxon test. A two-sided p $<0.05$ was considered significant.

Results: A total of 39 patients were enrolled, with a mean age of $62.1 \pm 14$ years and a male predominance (90\%). The most common causes of heart failure were ischemic and dilated cardiomyopathy. The mean ejection fraction was $30 \%$ and the median time of disease duration was 84 months (IQR 33-144). Patients who were enrolled for less than one month were excluded, with a total of 34 patients analyzed. Patients were followed in the TMP for a median of 320 days. The number of emergency department admissions was reduced by $66 \%$ ( $p<0.001$ ), and the number of hospitalizations for heart failure was reduced by $68 \%(p<0.001)$. The TMP had no impact on the number of hospitalizations for other causes.

Conclusions: This trial suggests that a TMP could reduce health service use in patients with heart failure.

Keywords: Heart Failure/physiopathology; Telemonitoring; Hospitalization; Emergency Services.

Full texts in English - http://www.arquivosonline.com.br

Correspondência: Isabel O. Cruz •

Hospital Pedro Hispano - R. de Dr. Eduardo Torres, Sra. da Hora Matosinhos 4464-513 - Portugal

E-mail: aisabelocruz@gmail.com

Artigo recebido em 26/11/2020, revisado em 15/03/2021, aceito em 24/03/2021

DOI: https://doi.org/10.36660/abc.20201264 


\section{Introdução}

A insuficiência cardíaca (IC) é um grande problema de saúde pública, cuja incidência tem aumentado, e com mortalidade e morbidade significativas. ${ }^{1}$ Em Portugal, estimase que a prevalência seja de $4,36 \% .^{2}$ A evolução natural dessa doença é a piora dos sintomas e a diminuição da capacidade funcional com o tempo, com episódios de descompensação aguda, que geralmente levam à internação hospitalar. Após a primeira admissão, até $50 \%$ dos pacientes são readmitidos no período de seis meses após a alta ${ }^{3}$ e entre $17 \%$ e $45 \%$ morrem durante o primeiro ano. ${ }^{4}$ Internações hospitalares repetidas por IC têm um impacto negativo no prognóstico, sendo um preditor independente de mortalidade. ${ }^{5}$

As principais estratégias para evitar a internação hospitalar são o tratamento farmacológico adequado, a educação do paciente, o acompanhamento estruturado e o automonitoramento. ${ }^{6}$ Várias estratégias foram tentadas para se identificar os sinais de deterioração clínica, permitindo que houvesse uma intervenção antes da exacerbação aguda. O telemonitoramento é o uso da tecnologia para monitorar remotamente os pacientes em casa. ${ }^{7}$ Ele foi proposto pela primeira vez nas Diretrizes da Sociedade Europeia de Cardiologia (European Society of Cardiology - ESC) de 2016 para o diagnóstico e tratamento de IC aguda e crônica, com recomendação de classe II, com nível de evidência B. ${ }^{6}$ Estudos demonstraram que programas de telemonitoramento poderiam reduzir os índices de internações hospitalares e admissões em serviços de emergência, durações dos períodos de internação, e até mesmo a mortalidade relacionada à IC. 8-11 Outras possíveis vantagens são o envolvimento dos pacientes e das famílias no controle da doença, a otimização do tratamento médico em tempo hábil, o aumento da adesão ao tratamento e a melhoria da qualidade de vida do paciente. ${ }^{9}$ No entanto, esses resultados são inconsistentes, dado que outros estudos apresentam resultados nulos. ${ }^{12,13}$ Isso provavelmente se deve às diferenças entre as populações estudadas, os sistemas de saúde, e os tipos de telemonitoramento. ${ }^{14}$

\section{Objetivos}

O objetivo deste estudo é avaliar o impacto do telemonitoramento remoto não invasivo em pacientes portugueses com insuficiência cardíaca avançada em internações hospitalares e admissões em serviços de emergência.

\section{Materiais e métodos}

\section{Desenho do estudo}

Este é um estudo retrospectivo observacional de antes e depois de pacientes cadastrados em um programa avançado de telemonitoramento (PTM) de insuficiência cardíaca. Isso significa que cada paciente tem seu próprio controle, comparando eventos no ano anterior à entrada no programa e durante o período de PTM.

\section{Seleção dos pacientes}

Para serem incluídos no PTM, os pacientes precisavam ter mais de 18 anos, ter diagnóstico de IC e ser capazes de lidar com os dispositivos médicos. Selecionamos dados sobre pacientes que se cadastraram no programa entre $1^{\text {o }}$ de janeiro de 2018 e 30 de novembro de 2019. Participantes acompanhados por menos de um mês foram excluídos da análise.

\section{Protocolo de telemonitoramento}

O processo de telemonitoramento consistiu em medições diárias de dados fisiológicos, especificamente peso corporal, pressão arterial, frequência cardíaca, saturação de oxigênio e temperatura corporal, além da realização de um eletrocardiograma de três derivações semanalmente.

Após a seleção, os pacientes e cuidadores passaram por treinamento sobre como usar os dispositivos e transmitir os dados. Os valores basais foram definidos como uma mediana dos três primeiros valores registrados. Os desvios que disparariam um alerta foram definidos pela equipe médica (Tabela 1).

Foram definidos dois tipos de alerta. Alertas técnicos aconteciam se houvesse falha na transmissão de dados ou se não houvesse relato de dados, e estes eram resolvidos por um técnico. Alertas clínicos foram uma medida acima ou abaixo dos limites pré-definidos. Um enfermeiro ligaria para os pacientes com alerta clínico para perguntar sobre os sintomas e aplicar o teste de Morisky-Green para medir a aderência aos medicamentos. Se o alerta clínico fosse validado, o médico responsável entraria em contato com o paciente para decidir sobre a melhor estratégia de controle.

Tabela 1 - Definição das variáveis relatadas e desvios que disparam um alerta

\begin{tabular}{|c|c|c|}
\hline & Normal & Alerta \\
\hline Saturação periférica de $\mathrm{O}_{2}\left(\mathrm{SpO}_{2}\right)$ & $\Delta<4 \%$ da basal & $\Delta \geq 4 \%$ e $\mathrm{SpO}_{2}<92 \%$ \\
\hline Frequência Cardíaca & $50-100$ ppm & $<50$ ou $>100$ \\
\hline Pressão arterial sistólica & $\Delta \leq 20 \%$ da basal & $\Delta>20 \%$ da basal \\
\hline Pressão arterial diastólica & $\Delta \leq 20 \%$ da basal & $\Delta>20 \%$ da basal \\
\hline Peso corporal & $\Delta<1 \mathrm{Kg}$ & $\Delta \geq 1 \mathrm{Kg}$ em $24 \mathrm{~h}$ ou $\geq 2 \mathrm{Kg}$ em 3 dias \\
\hline Temperatura & $\leq 37,5^{\circ} \mathrm{C}$ & $>37,5^{\circ} \mathrm{C}$ \\
\hline Eletrocardiograma de três derivações & FC $50-100$ & $\mathrm{FC}<50$ ou $>100$ \\
\hline
\end{tabular}

FC: Frequência cardíaca. Kg: quilograma. 


\section{Coleta de dados}

Dados demográficos dos pacientes e características da doença foram obtidos de prontuários médicos eletrônicos. Coletamos dados sobre internação hospitalar global e por insuficiência cardíaca, bem como admissões em serviços de emergência, do ano anterior à entrada no programa e ao período de cadastro.

\section{Análise estatística}

Os dados de linha de base foram resumidos utilizando-se estatísticas descritivas: médias e desvio padrão para dados contínuos com distribuição normal, medianas e faixas interquartis para dados enviesados, e porcentagens para dados categóricos. O teste de Shapiro-Wilk foi usado para determinar a normalidade da distribuição. A análise comparativa entre o cadastramento antes e depois do programa foi feita utilizandose o teste de Wilcoxon. Um p-valor bilateral de $<0,05$ foi considerado significativo. Todas as análises foram realizadas utilizando-se o software de análise de dados e estatística (SPSS).

\section{Resultados}

Um total de 39 pacientes foram incluídos no programa. As características da linha de base são apresentadas na tabela 2. A média de idade foi de 62 anos, variando entre 34 e 90 anos de idade. Houve uma predominância de pacientes do sexo masculino. A maioria dos pacientes tinha cardiomiopatia isquêmica ou dilatada, com uma duração mediana da doença de 84 meses (FIQ: 33-144). A fração de ejeção ventricular esquerda média foi de $29 \% \pm 9,3 \%$ e nenhum paciente apresentou fração de ejeção preservada. A fibrilação atrial estava presente em mais de dois terços dos pacientes.

Os pacientes foram acompanhados por um período mediano de 320 dias (FIQ: 166-486). Durante esse período, três pacientes abandonaram o estudo, um foi submetido a um transplante cardíaco, e cinco pacientes faleceram enquanto estavam no programa. As causas de morte foram a piora da IC em dois pacientes e infecção em três outros casos. Para esses sujeitos, todos os eventos que antecederam ao momento da interrupção foram levados em conta na análise principal. Os demais 25 sujeitos ainda estavam cadastrados no PTM no momento da análise.

A adesão foi boa, com $58 \%$ dos pacientes relatando todos os dados pelo menos $75 \%$ do tempo, e $75 \%$ dos pacientes relatando pelo menos um parâmetro mais de $75 \%$ do tempo.

Houve um total de 2928 alertas clínicos, porém apenas 31 foram confirmados como sendo clinicamente relevantes, especialmente as mudanças no peso e na frequência cardíaca. Os $98,9 \%$ restantes dos alertas foram considerados não significativos devido à ausência de sintomas ou medições incorretas. Os alertas significativos são definidos como leves, moderados ou graves, de acordo com o desvio dos limites pré-definidos. Nesses casos, o médico ligaria para o paciente e decidiria sobre o controle, conforme ilustrado na figura 1. Metade dos pacientes foram atendidos no departamento de emergência; entretanto, os demais casos foram resolvidos por alterações terapêuticas, uma consulta na clínica da IC, ou apenas o monitoramento.
Tabela 2 - Características de linha de base da população

\begin{tabular}{|c|c|}
\hline & $n=34^{*}$ \\
\hline Idade, anos & $62 \pm 14$ \\
\hline Sexo masculino, $\mathbf{n}(\%)$ & $30(88 \%)$ \\
\hline \multicolumn{2}{|l|}{ Etiologia, n (\%) } \\
\hline Doença cardíaca isquêmica & $13(38,3 \%)$ \\
\hline Cardiomiopatia dilatada & $14(41,2 \%)$ \\
\hline Relacionada ao consumo de álcool & $3(8,9 \%)$ \\
\hline Idiopática & $7(20,7 \%)$ \\
\hline Pós-quimioterapia & $1(2,9 \%)$ \\
\hline Pós-miocardite & $2(5,8 \%)$ \\
\hline Familiar & $1(2,9 \%)$ \\
\hline Cardiomiopatia hipertrófica & $3(8,9 \%)$ \\
\hline Miocárdio não compactado de ventrículo esquerdo & $1(2,9 \%)$ \\
\hline Doença cardíaca congênita & $1(2,9 \%)$ \\
\hline Displasia arritmogênica do ventrículo direito & $1(2,9 \%)$ \\
\hline Amiloidose & $1(2,9 \%)$ \\
\hline \multicolumn{2}{|l|}{ Fração de ejeção ventricular esquerda, n (\%) } \\
\hline Normal $(>50 \%)$ & $0(0 \%)$ \\
\hline Disfunção leve (40-50\%) & $8(23,5 \%)$ \\
\hline Disfunção moderada (30-40\%) & $9(26,5 \%)$ \\
\hline Disfunção grave (<30\%) & $17(50 \%)$ \\
\hline \multicolumn{2}{|l|}{ Classe NYHA, n (\%) } \\
\hline I & $0(0 \%)$ \\
\hline II & $15(44,1 \%)$ \\
\hline III & $18(53 \%)$ \\
\hline IV & $1(2,9 \%)$ \\
\hline Fibrilação atrial, n (\%) & $22(64,7 \%)$ \\
\hline \multicolumn{2}{|l|}{ Medicamentos, n (\%) } \\
\hline Betabloqueador & $30(88,2 \%)$ \\
\hline Inibidores da ECA ou BRA & $12(35,3 \%)$ \\
\hline Inibidores da NRA & $15(44,1 \%)$ \\
\hline Antagonista do receptor da aldosterona & $31(91,2 \%)$ \\
\hline
\end{tabular}

NYHA: New York Heart Association; ECA: enzima conversora da angiotensina; BRA: bloqueador do receptor de angiotensina; NRA: neprilisina receptor de angiotensina. * Dados apresentados como média \pm desvio padrão ou $n$, (\%)

O número total de consultas em serviços de emergência diminuiu de 100 admissões, no ano anterior ao cadastro, para 34, durante o programa de telemonitoramento (redução de $66 \%, p<0,001)$. As internações hospitalares por IC diminuíram de 71 para $23(68 \%, p<0,001)$ e o número de dias no hospital também diminuiu significativamente, de 692 dias para 178 dias. Não foram encontradas diferenças nas admissões devido a outras causas. Esses resultados estão ilustrados na figura 2 .

Nenhum evento adverso foi causado pelo sistema de monitoramento. 


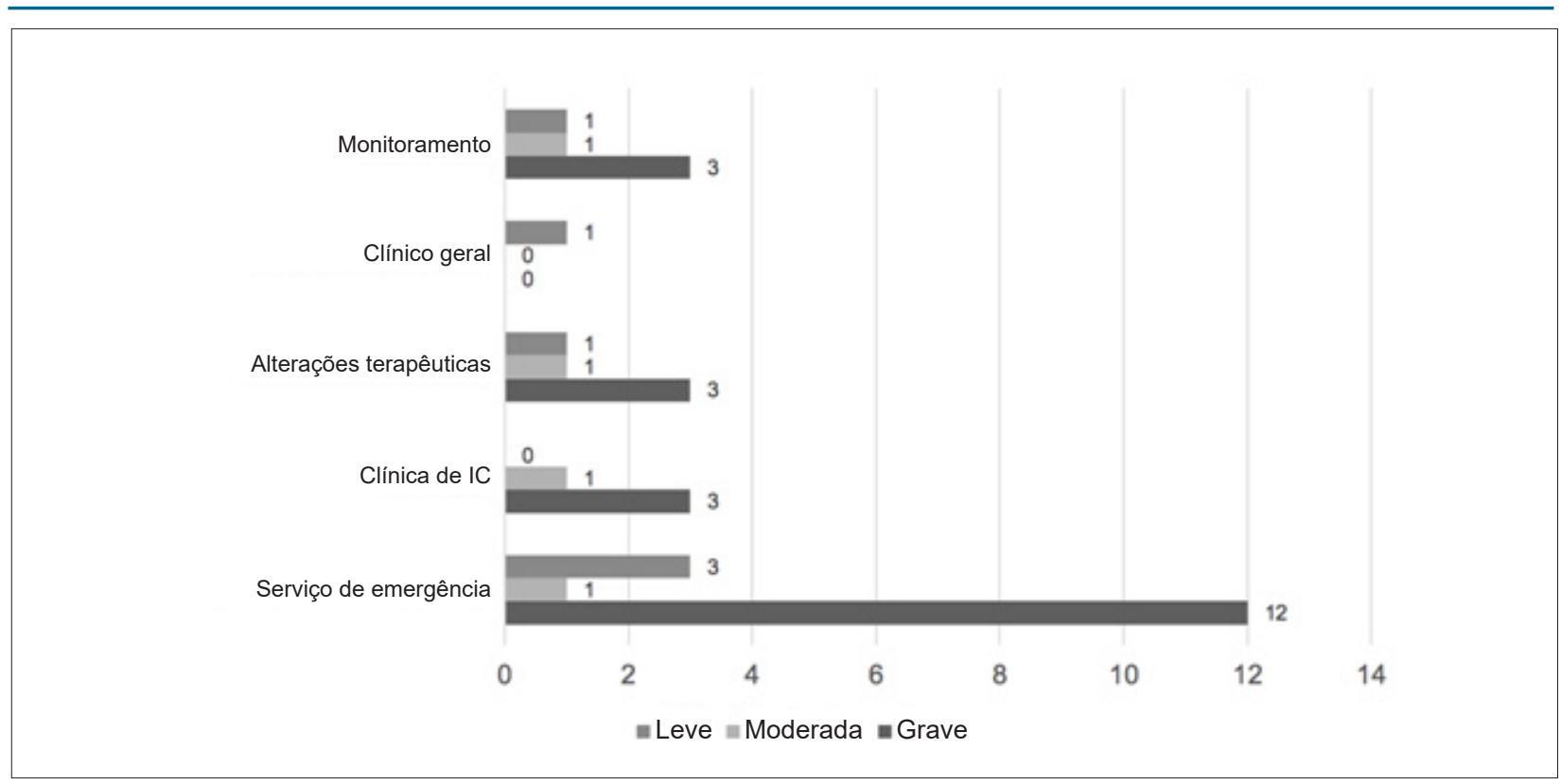

Figura 1 - Gerenciamento da decisão médica após alerta clínico relevante e contato telefônico com o paciente.

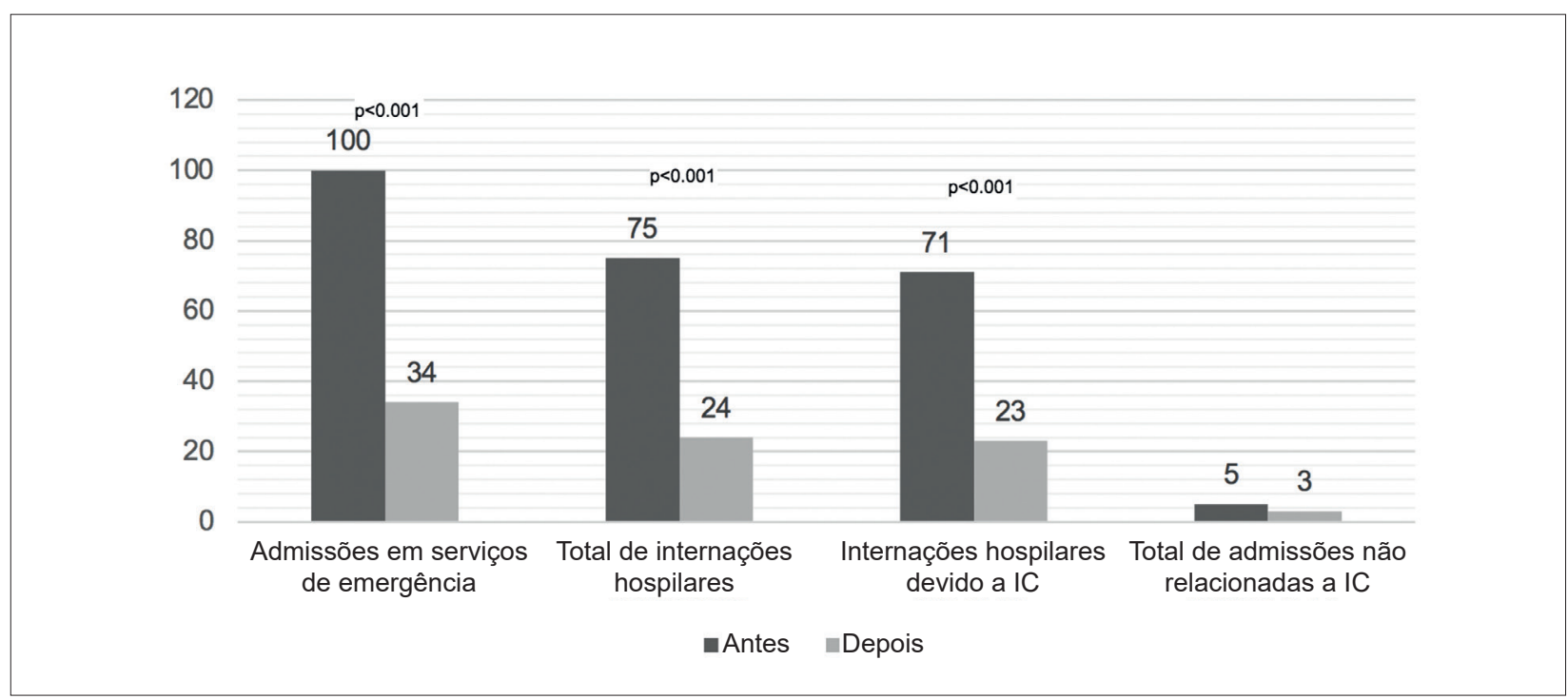

Figura 2 - A análise comparativa pelo teste de Wilcoxon revelou redução significativa nas admissões em pronto-socorro e nas internações por IC, ao comparar os números do ano anterior com os números durante a adesão ao programa de telemonitoramento.

\section{Discussão}

Recentemente, o telemonitoramento domiciliar tem aparecido como uma opção adicional no controle de IC, disponibilizando sinais vitais e sintomas regulares e confiáveis de pacientes da comunidade. Este é o primeiro estudo a testar essa hipótese na população portuguesa. Encontrouse uma redução significativa de $68 \%$ nas internações hospitalares devido à piora da IC e uma redução de $66 \%$ em consultas globais em serviços de emergência. Outro ponto importante é a redução do período de estadia no hospital, provavelmente resultante tanto da identificação precoce da descompensação e da alta antecipada, com o monitoramento próximo do paciente. Isso é particularmente relevante devido à limitação de leitos hospitalares. Esses resultados corroboram estudos anteriores que demonstraram os benefícios do telemonitoramento remoto.

O monitoramento diário do peso é uma recomendação de classe I no controle da $\mathrm{IC}^{6}$, já que a retenção de líquidos é um sinal de piora e não adesão ao tratamento diurético. Em nosso estudo, esse foi o alerta clínico mais comumente significativo identificado, levando a uma intervenção, principalmente alterações terapêuticas, consultas clínicas, ou 
admissões em serviços de emergência. Entretanto, Zhang et al., ${ }^{15}$ demonstraram que a pesagem isoladamente tinha valor limitado.

Em 2019, os programas de telemonitoramento foram endossados ainda mais pela Sociedade Europeia de Cardiologia (ESC), ${ }^{16}$ principalmente devido a duas publicações. O estudo TIM-HF $2^{17}$ demonstrou que uma avaliação domiciliar regular de peso, pressão arterial, frequência cardíaca, saturação de oxigênio, eletrocardiograma e status geral da saúde poderia reduzir a proporção de dias de afastamento devido a internações hospitalares por problemas cardiovasculares (especialmente IC) não planejadas ou mortes $(p=0,046)$, bem como mortalidade global (FC 0,70; $p=0,028$ ). Uma análise de Cochrane ${ }^{18}$ de 25 estudos concluiu que o telemonitoramento reduziu a mortalidade global em $20 \%$, e a internação hospitalar por IC em 30\%.

A ESC afirmou que o protocolo usado no estudo TIMHF2 deveria ser tentado em outros países, para testar sua capacidade de ser reproduzido. ${ }^{16} \mathrm{~A}$ população participante do estudo TIM-HF2 foi similar à nossa em termos de distribuição por sexo, classe da NYHA, e etiologia da insuficiência cardíaca. Entretanto, sua população era 8 anos mais velha, e $25 \%$ de seus pacientes apresentavam fração de ejeção preservada, enquanto, em nosso estudo, não havia nenhum. O programa de telemonitoramento é muito semelhante ao nosso e nossos resultados positivos podem indicar que ele seja um método efetivo de monitoramento remoto.

O controle do paciente à distância não deve se limitar ao monitoramento dos sinais vitais. A equipe médica pode usar esses dados para individualizar o tratamento, oferecer educação ao paciente, e introduzir ou aumentar terapias que modificam a doença. Essa abordagem pode levar a um impacto mais significativo no prognóstico.

Em outros estudos, o grau de adesão variou de $80 \%$ a $90 \%, 7,14$ que é um valor melhor que nossos números. Isso se deve provavelmente aos fatos de que os pacientes apresentaram menos adesão na prática clínica diária se comparado aos momentos de estudos clínicos. Em Portugal, conforme mostrado pelo HLS-EU-PT, ${ }^{18} 61 \%$ da população pesquisada têm um nível de conhecimento de saúde geral inadequado. Isso pode ser uma barreira para um tratamento eficaz da doença, já que esses pacientes têm mais dificuldade de entender a doença e seu controle. ${ }^{19}$ Alguns estudos também demonstraram que isso leva a uma baixa adesão aos medicamentos e ao aumento das internações hospitalares. Ao entrar nesse tipo de programa, os pacientes e cuidadores recebem mais conhecimento e responsabilidade sobre o controle da doença. ${ }^{20}$ Isso provavelmente ajuda a explicar por que, embora o número de alertas clínicos significativos seja baixo, o número de internações hospitalares por IC diminuem grandemente.

Alguns estudos demonstraram que a idade não tem impacto nesses resultados, com pacientes acima de 75 anos de idade tendo o mesmo benefício que os pacientes mais jovens. ${ }^{21}$ Isso é importante uma vez que temos uma população em envelhecimento, e com alta prevalência de IC e internações hospitalares frequentes.

As principais limitações deste estudo são aquelas associadas ao estudo antes e depois, principalmente a ameaça da evolução, que é definida como outros eventos que poderiam afetar os resultados. Nesse tipo de estudo, outras variáveis, como as alterações nos medicamentos ou outras intervenções, não são registradas. Também temos uma amostra pequena. Entretanto, este estudo ainda pode gerar evidência preliminar para a eficácia da intervenção em uma população com insuficiência cardíaca relativamente grave. Outra limitação é que as internações hospitalares que ocorrem em hospitais fora do Serviço Nacional de Saúde não foram registradas, embora não sejam incomuns.

Pesquisas posteriores devem se concentrar na identificação dos parâmetros biológicos mais importantes a serem monitorados, na definição dos subgrupos de pacientes que serão mais beneficiados por essa abordagem, e de quais são os programas com melhor relação custobenefício.

\section{Conclusões}

Nosso programa de telemonitoramento não invasivo reduziu as internações hospitalares e as admissões em serviços de emergência devido a IC, bem como o número de dias de internação por IC. A implementação de um programa desse tipo deve ser considerada para a melhoria dos resultados para pacientes com insuficiência cardíaca.

\section{Contribuição dos autores}

Concepção e desenho da pesquisa: Cruz IO, Costa S, Franco F, Gonçalves L; Obtenção de dados e Redação do manuscrito: Cruz IO; Análise e interpretação dos dados e Análise estatística: Cruz IO, Teixeira R; Revisão crítica do manuscrito quanto ao conteúdo intelectual importante: Costa S, Teixeira R, Franco F, Gonçalves L.

\section{Potencial conflito de interesse}

Não há conflito com o presente artigo

\section{Fontes de financiamento}

O presente estudo não teve fontes de financiamento externas.

\section{Vinculação acadêmica}

Não há vinculação deste estudo a programas de pósgraduação.

\section{Aprovação ética e consentimento informado}

Este artigo não contém estudos com humanos ou animais realizados por nenhum dos autores. 


\section{Referências}

1. Savarese G, Lund LH. Global public health burden of heart failure. Card Fail Rev. 2017;3(1):7-11. https://doi.org/10.15420/cfr.2016:25:2.

2. Ceia F, Fonseca C, Mota T, Morais H, Matias F, Sousa A, et al. Prevalence of chronic heart failure in Southwestern Europe: the EPICA study. Eur J Heart Fail. 2002;4(4):531-539. https://doi.org/10.1016/s1388-9842(02)00034-x.

3. Giamouzis G, Kalogeropoulos A, Georgiopoulou V, Laskar S, Smith AL, Dunbar S, et al. Hospitalization epidemic in patients with heart failure: risk factors, risk prediction, knowledge gaps, and future directions. J Card Fail. 2011;17(1):54-75. https://dor.org/ 10.1016/j.cardfail.2010.08.010.

4. Ponikowski P, Anker SD, AlHabib KF,Cowie MR, Force TL. Hu S, et al. Heart failure: preventing disease and death worldwide. ESC Heart Failure. 2014;1(1):4-25. https://doi.org/10.1002/ehf2.12005.

5. Bello NA, Claggett B, Desai AS, McCurray JV, Ganger C, Yusef S, et al. Influence of previous heart failure hospitalization on cardiovascular events in patients with reduced and preserved ejection fraction. Circ Heart Fail. 2014;7(4):590595. https://doi.org/10.1161/CIRCHEARTFAILURE.113.001281.

6. Ponikowski P, Voors AA, Anker SD,Bueno E, Cleland JF, Costa I. et al. 2016 ESC Guidelines for the diagnosis and treatment of acute and chronic heart failure: The Task Force for the diagnosis and treatment of acute and chronic heart failure of the European Society of Cardiology (ESC). Eur Heart J.2016;37(27):2129-200. https://doi.org/10.1093/eurheartj/ehw128.

7. Cleland JG, Louis AA, Rigby AS, Janssens U, BalkAH; TEN-HMS Investigators. Noninvasive home telemonitoring for patients with heart failure at high risk of recurrent admission and death: the Trans-European NetworkHome-Care Management System (TEN-HMS) study. J Am Coll Cardiol. 2005;45(10):1654-64. https://doi.org/10.1016/j.jacc.2005.01.050.

8. Giamouzis G, Mastrogiannis D, Koutrakis K, Karayanis G, Parisis C, Rountas $\mathrm{C}$, et al. Telemonitoring in chronic heart failure: a systematic review. Cardiol Res Pract. 2012;2012:410820. https://doi.org/10.1155/2012/410820.

9. Inglis SC, Clark RA, McAlister FA, et al. Structured telephone support or telemonitoring programmes for patients with chronic heart failure. Cochrane Database Syst Rev. 2010;8:CD007228. https://do.org/:10.1002/14651858. CD007228.pub2.

10. Louis AA, Turner T, Gretton M, Baksh A, Cleland JG. A systematic review of telemonitoring for the management of heart failure. Eur J Heart Fail. 2003;5(5):583-90. https//doi.otg/ 10.1016/s1388-9842(03)00160-0.

11. Koehler F, Winkler S, Schieber M, Sechtem U, Stangl K, Bohm M, et al. Telemedical Interventional Monitoring in Heart Failure (TIM-HF), a randomized, controlled intervention trial investigating the impact of telemedicine on mortality in ambulatory patients with heart failure: study design. Eur J Heart Fail. 2010;12(12):1354-62. https://doi.org/10.1093/ eurjhf/hfq199.
12. Chaudhry SI, Mattera JA, Curtis JP,Spertus JA, Herrin J, Lin Z, et al. Telemonitoring in patients with heart failure [published correction appears in N Engl J Med. 2013 Nov 7;369(19):1869]. N Engl J Med. 2010;363(24):2301-09. https://doi.org/10.1056/NEJMoa1010029.

13. Ong MK, Romano PS et al. Effectiveness of Remote Patient Monitoring After Discharge of Hospitalized Patients With Heart Failure: The Better Effectiveness After Transition-Heart Failure (BEAT-HF) Randomized Clinical Trial. JAMA Intern Med. 2016;176(3):310-18. https://doi.org/10.1001/ jamainternmed.2015.7712

14. Dar O, Riley J, Chapman C, Edgington S, Auerbach AD, Black KT,et al. A randomized trial of home telemonitoring in a typical elderly heart failure population in North West London: results of the Home-HF study. Eur J Heart Fail. 2009;11(3):319-25. https://doi.org/10.1093/eurjhf/hfn050.

15. Zhang J, Goode KM, Cuddihy PE, Cleland JG. TEN-HMS Investigators Predicting hospitalization due to worsening heart failure using daily weight measurement: analysis of the Trans-European Network-Home-Care Management System (TEN-HMS) study. Eur J Heart Fail. 2009;11(4):420-7. https://doi.org/10.1093/eurjhf/hfp033.

16. Seferovic PM, Ponikowski P, Anker SD, Bauersachs I, Chiocell O, Cleland JG, et al. Clinical practice update on heart failure 2019: pharmacotherapy, procedures, devices and patient management. An expert consensus meeting report of the Heart Failure Association of the European Society of Cardiology. Eur J Heart Fail. 2019;21(10):1169-1186. https://doi. org/10.1002/ejhf.1531

17. Koehler F, Koehler K, Deckwart O, et al. Efficacy of telemedical interventiona management in patients with heart failure (TIM-HF2): a randomised, controlled, parallel-group, unmasked trial. Lancet. 2018;392(10152):104757. https://doi.org/ 10.1016/S0140-6736(18)31880-4

18. Inglis SC, Clark RA, Dierckx R, Prieto-Merino D, Cleland JG. Structured telephone support or non-invasive telemonitoring for patients with heart failure. Cochrane Database Syst Rev. 2015;10:CD007228. https://doi. org/10.1002/14651858.CD007228.pub3.

19. Pedro AR, Amaral O, Escoval A. Literacia em saúde, dos dados à ação: Tradução, validação e aplicação do European Health Literacy Survey em Portugal. Rev Portuguesa Saúde Pública. 2016;34(3):259-75. http://dx.doi. org/10.1016/j.rpsp.2016.07.002.

20. Koulaouzidis G, Barrett D, Mohee K, Clark AL. Telemonitoring in subjects with newly diagnosed heart failure with reduced ejection fraction: From clinical research to everyday practice. J Telemed Telecare. 2019;25(3):16771. https://doi.org/10.1177/1357633X17751004.

21. Nakamura N, Koga T, Iseki H. A meta-analysis of remote patient monitoring for chronic heart failure patients. J Telemed Telecare. 2014;20(1):11-7. https://doi.org/ 10.1177/1357633X13517352. 